\title{
Assessing the Cartographic Visualization of Moving Objects
}

\author{
Biadgilgn, D.M ${ }^{1^{*}}$, Blok, C.A ${ }^{2}$ and Huisman, $\mathrm{O}^{2}$ \\ ${ }^{1 *}$ Department of Geography and Environmental Studies, College of Social Science and \\ Languages, Mekelle University, P.O.Box: 231, Mekelle, Ethiopia (biadgilgnd@gmail.com) ${ }^{*}$. \\ ${ }^{2}$ Department of Geo-Information Processing (GIP), Faculty of Geoinformation Science and Earth \\ Observation (ITC), University of Twente, P.O.Box: 217, 7500, AE Enschede, Netherlands.
}

\begin{abstract}
Nowadays, there is a lot of interest in studying dynamic spatial phenomena. There are various dynamic phenomena in the world among which moving objects are worth exemplifying. Recently, moving objects are getting attention in database applications and in visualization. Moving objects are of two categories: individual moving objects and group moving objects. As visualization is important for various human activities like navigation, planning, monitoring, and academic accomplishments, the selection of the appropriate visualization method for a particular application is important. It is, however, not always known what 'appropriate' is in a particular context. This research is concerned with individual moving objects and their movement behavior. The research focused on visualization of the trajectory movement characteristics of these individual moving objects. The main goal of this research is to find the optimal representation for visualizing moving objects (more precisely: walking people). Four representations are considered in this research: the single static map, multiple static maps, animation, and the space-time cube. The study is conducted by considering four movement characteristics (or aspects of moving objects): speed change, returns, stops, and path of movement. The ability of users to perceive and understand these aspects from the four representation methods is studied. To achieve the goal, a user test is conducted using the questionnaire method (the users performing tasks). The visualizations were prepared on three levels of complexity - the complexity varying in the number of objects represented and the geometry of the trajectory along which they move (Level 1 with one object and simplest in complexity of geometry of the trajectory; Level 2 with two objects and more complex geometry of the trajectory; and Level 3 with three objects and most complex geometry of the trajectory). A prediction about suitability was made based on theory, cartographic knowledge, and common sense. The prediction shows that animation is better than the other methods for visualizing returns and speed change at all levels, and path at Level 2. In the other aspects and levels of complexity animation had equal suitability with the other methods. However, the prediction is not enough to make the selection of the best method for a particular use. Thus, user test is needed. The result of the suitability after test illustrates that users perceive and understand the movement characteristics better in an animation than in the other representations, at different levels of complexity. Animation is most suitable for stops at Level 1, for returns at Level 3, and for speed change at Level 1 . In the rest, animation has equal suitability with one or more of the other methods, except for path at Level 2 where the space-time cube is most suitable. In the overall result, therefore, animation is found to be the optimal representation for moving objects (walking people).
\end{abstract}

Key words: Change, Movement, Moving objects, Trajectory, Visualization, Single static map, Multiple static maps, Animation, Space-time cube 


\section{INTRODUCTION}

Visualization through graphic representations has been an effective way to communicate with both abstract and concrete ideas. Its application is expanding in many disciplines, for example, in cartography, engineering and medicine. The use of graphics makes communication and analysis easy. In a geo-visualization environment, graphics (like maps) help to visually understand relationships, patterns, trends, and movements in a number of ways, for instance using multiple representations without constraints (Kraak and Ormeling 2003). The representation of spatiotemporal phenomena in graphic visualization consists of three important components: time, space, and attribute. Time is receiving increasing attention in geo-visualization environments; without it the study of spatiotemporal process, changes or events cannot be successful (Kraak and Ormeling 2003). The most commonly used spatiotemporal visualization techniques are static maps (single and multiple), animated maps and the space-time cube (STC).

The use of static maps is one of the earliest applications of geo-visualization. In single static maps, graphic variables (position, form, orientation, color, size, etc) are used to indicate changes in time or represent an event at a certain time moment. In the temporal representations perspective, small multiples display a set of maps, each for different events in time and change therefore, is depicted in successive snapshots (Slocum 1999). In small multiples, the temporal sequence is represented by a spatial sequence for the user to perceive the variation in time (Kraak and Ormeling 2003; de By et al. 2004). Computer animations have become important tools in the cartographic application in recent times. Animations are all about changes (in locations, attributes and/or time) (Kraak 1999). They show relationships and correlations between geographic phenomena and time (Karl 1992; Ogao 2002). Animations are conceived to be important, e.g. in monitoring activities, in account of detection of change and perception of spatiotemporal patterns and trends of the represented dynamic object (Blok 2005). An important concept in Time-Geography is also the space-time cube where space is represented along the $\mathrm{x}$ and $\mathrm{y}$ axis and time is represented along the $\mathrm{z}$ axis (Kraak and Koussoulakou 2004). It is a 3D representation of movement, or paths where the user can visualize changes, durations, and other aspects of paths in time of, for example, people, cars, or animals. The classical space-time cube studies deal with the behavior of humans considering certain activities of individuals like following a path through space and time (Xia and Kraak 2005). Kraak and Koussoulakou (2004) 
stated that a better exploration and understanding of temporal events in the geo-world requires the integration of geo-visualization with the space-time cube.

As regards the relative effectiveness of representation of spatiotemporal geographic objects and acquiring knowledge about these objects from static maps, animation, and the space-time cube, experts have significantly different views. Blok (2005), among others, has raised a question as to whether animations are effective media for visualization. Some argued that animated maps might be better than static maps and others contended to the contrary based on the use of context. Cartographic animations show process directly and static maps hardly show dynamic aspects of reality (Karl, 1992). To show changes in time and space, multiple still images can be placed on a page but they may become too small and consequently, difficult to compare (Dorling and Openshaw 1994). Dorling and Openshaw (1994) deducted that changes and patterns can be shown on a page in a sequence of static maps on condition that the dynamic information is extremely simple. Temporal animation has fascinating advantages over temporal static maps (series) particularly in depicting real world process and trends, and in explaining spatial relationship (Kraak and Ormeling 2003).

On the other hand, Tversky (2002) contended that the recently undertaken experiments failed to show the effectiveness of animations over static maps for learning on the reason that animations lack equivalent and comparable contents. They also mentioned that complexity of the content in (non-interactive) animations challenges comprehension. According to Kim and Yoon (2007), animations are not more effective than equivalent static graphics in learning for low cognition (comprehension) related learners. Fabrikant (2005) mentioned that experimental researches in many fields have shown that static graphics facilitate comprehension and communication of complex phenomena.

Moreover, earlier geo-visualization studies (Kraak, 2003; Kristensson, et al, 2007; Weghe, 2004)) explained that the space-time cube representation is powerful in revealing complex spatiotemporal patterns of moving objects, for the fact that time and spatial information are displayed simultaneously. Kraak (2003) explained that the space-time cube is most suitable for the display and analysis of paths of phenomena where time is represented in the third dimension. It can also be applicable for real time monitoring, for example, running races. Kristensson et al (2007), however, argued that the actual usefulness of the space-time cube representation in revealing complex spatiotemporal patterns has not been empirically validated. They further 
asserted in their investigation by comparing the space-time cube and baseline 2D representations that the space-time cube is at least worth further investigation, for example by varying data density (complexity), choice of maps, domains or level of expertise.

There are significant researches done on static maps and animations and few on the Space-Time Cube that discuss on their effectiveness in representing dynamic phenomena in various fields (Tversky, Morrison et al. 2002; Blok, 2005; Fabrikant, 2005; Fabrikant and Goldsberry, 2005; Opach, 2007). However, there are contradictions and doubts as to the suitability of static maps, animations, and/or the space-time cube in terms of understanding, communication, and geographic knowledge acquisition. Evaluation of static maps, animations, and/or the space-time cube could best be carried out based on the purpose, user tasks, and characteristics of the data. The possibility of extracting relevant information and discovering geospatial knowledge can be important measures of effectiveness (Blok, 2005). The degree users detect and comprehend change, understand spatiotemporal patterns, identify important geospatial objects and their attributes explain the effectiveness of cartographic representations. The problem is that there is hardly clearly established knowledge (but many doubts and questions) as to whether static maps, animation, and/or the space-time cube is/are optimal in the visualization of dynamic phenomena. To fill this gap of knowledge (Kumar, 2005), some researchers come up with results that make animations superior to the other cartographic representations, while there are arguments otherwise as well. The question addressed here is which representation (single static map, multiple static maps, animation, and/or the space-time cube) can be considered optimal in the visualization of moving objects?

These issues form the prime impetus for this research. This research aims at narrowing the gap in knowledge by representing moving objects in single static map, animation, and the space-time cube to find which method is optimal for moving objects (with an application involving walking people). The research is conducted at different levels of complexity (in terms of number of objects and the geometry of the trajectory) of the objects represented. Speed change, returns, stops and path of movement are subject for investigation. The ability of the users to perceive and understand these aspects from different levels of complexity is the consideration on which the assessment is undertaken. It will be of assistance, in addition to determining the optimal representation method, in terms of illustrating the influence of the level of complexity in the visualization of moving objects. 


\section{MATERIALS AND METHOD}

\subsection{Design and Implementation}

The investigation of the suitability of visualizations to represent moving objects demands a clear and explainable methodological structure. Their movement characteristics are determined from literature (Andrienko and Andrienko, 2007; Blok, 2005; Meratnia and de By, 2004). The design is undertaken in such a way that makes clear (as much as possible) the movement characteristics of the objects (walking people). The representations are designed without interactive interface, but a minimum interactive environment is given to animation and the video version of the spacetime cube (the video version of the space-time cube enables the observers to see in different views). Data are prepared fictitiously. They are prepared in such a way that they would maintain the movement characteristics determined to be represented and suit the design of the representations.

\subsection{How the Visualizations Work}

All the representations are designed to have three levels of complexity, using the datasets of level one, level two and level three, prepared with one object, two objects and four objects respectively. The level of complexity not only varies with the number of objects represented, but also with the geometry of the trajectory along which the objects move. Even though the same data are used for all the representations, the representation of aspects such as speed changes, or returns might not be the same. For example, it is difficult to represent stops in single static maps. For distinguishing between the moving objects, they are given different colors. At the first level of complexity, where there is only one object, it is given a green color. At the second level of complexity, the two objects are colored red and blue, and at the third level of complexity, the four objects are colored red, blue, green, and yellow. Red, blue, and green are used repeatedly because they are easy to distinguish them on the grey background. . To exploit the potential of the representations in depicting spatio-temporal behaviors of moving objects, every possibility is attempted. For example, animation is accompanied by growing path; and the space-time cube is prepared with foot prints, grids for space and time indication, and it is also provided to users as a video to enable the users to see different views. 


\subsection{Test and Analysis}

In this phase, a user test is conducted to evaluate the suitability of static maps, animation, and the space-time cube in different levels of complexity. The users were kept in controlled room where they were provided with the representations. To fill their perception, understanding and acquisition of spatial knowledge, questionnaires were provided.

\subsection{The Representations}

\subsubsection{Single Static Maps}

In the single static maps, the three aspects (speed change, return, and path) were represented in the three levels of complexity (see Figures 2, 3 and 4). The paths of the movement of each object are displayed by line symbols so that the routes of movement can be easily depicted. To represent speed change, point symbols are used along the path at regular intervals. This will help visualize speed change from the distance travelled in that time interval. In order to reduce confusion, different symbols are used for 'start', 'return' and 'end' (see the legend in Figure 1). For each object, the start time is given to show, for example, which object arrives first at a certain location.

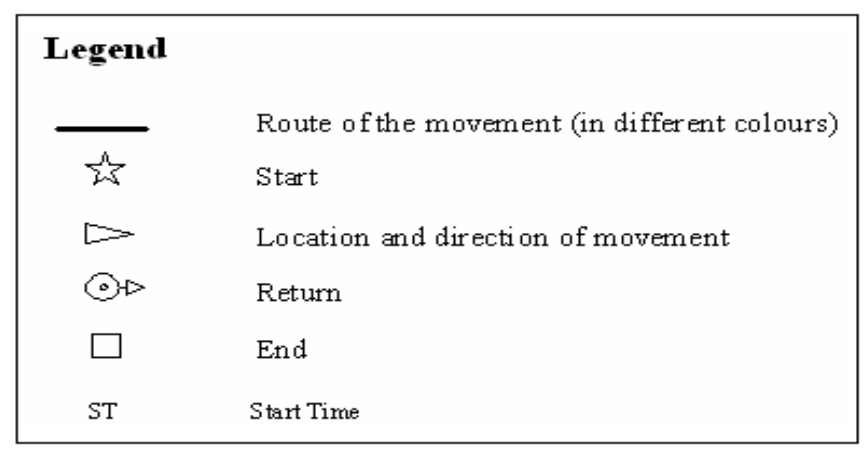

Figure 1 Legends used in static maps.

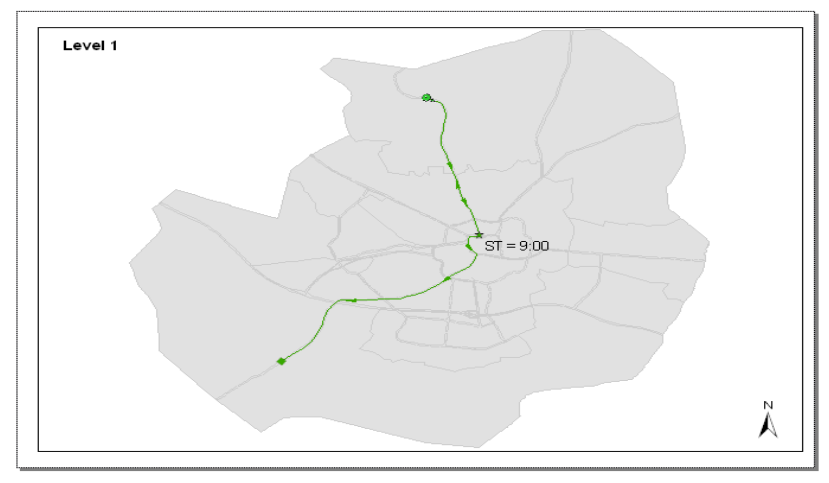

Figure 2. The first level of complexity for the single static maps with one moving object 


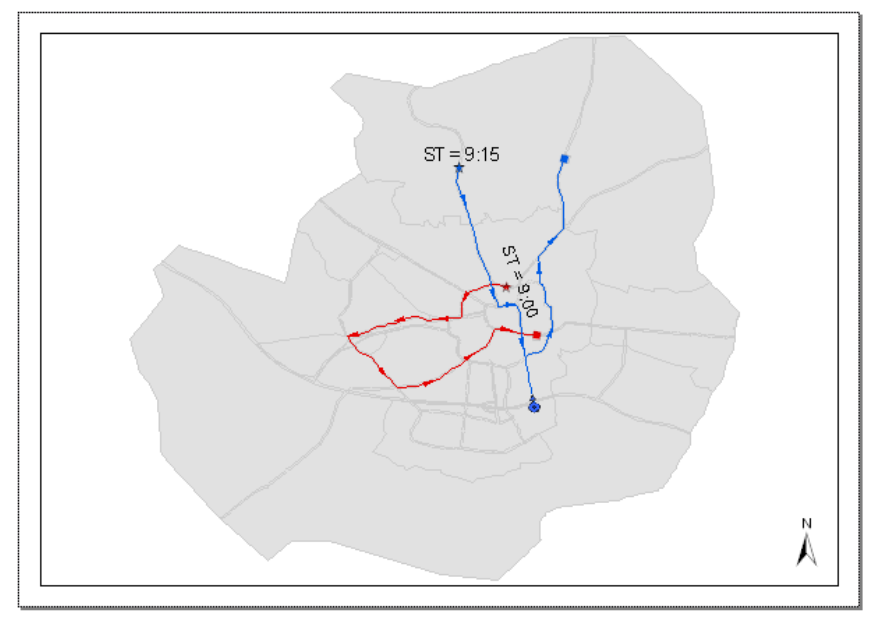

Figure 3. The second level of complexity for the single static maps with two moving objects

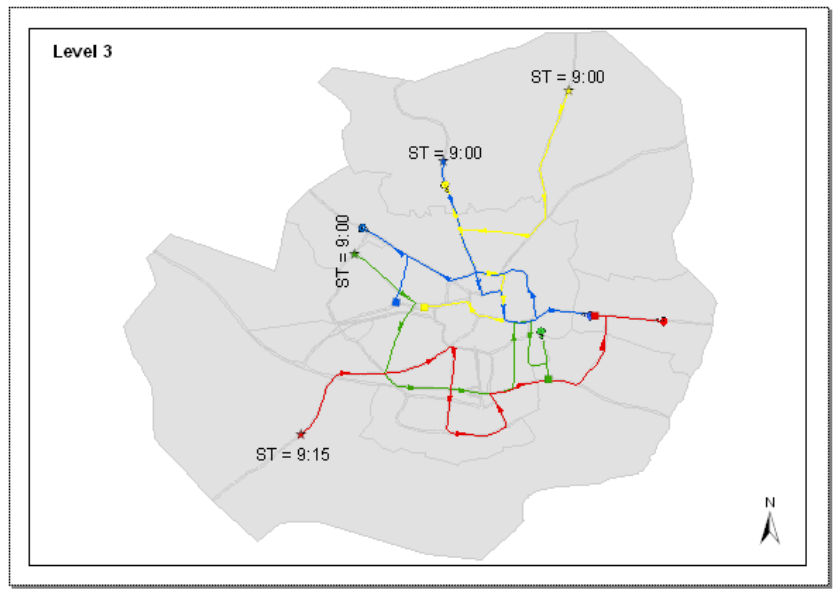

Figure 4. The third level of complexity for the single static maps with four moving objects

\subsubsection{Multiple Static Maps}

For the multiple static maps, the start and end symbol together with the symbols for location and direction are used. The loop symbol for return is not used because return can be visualized from the previous snapshot. The start time is also given on the first snapshot. In multiple static maps, all the aspects are represented at three levels of complexity (See Figures 5, 6 and 7). Speed change can be visualized by distance travelled between two snapshots and by comparing with the previous map (similar to the single static maps). Returns can be visualized by the location of the object and the direction indicated by the triangle. Stops can be represented by the location of the object on the same location in more than one snapshot. The trajectories of movement grow from the start up to the end. So on the last snapshot, the total path travelled is displayed. 


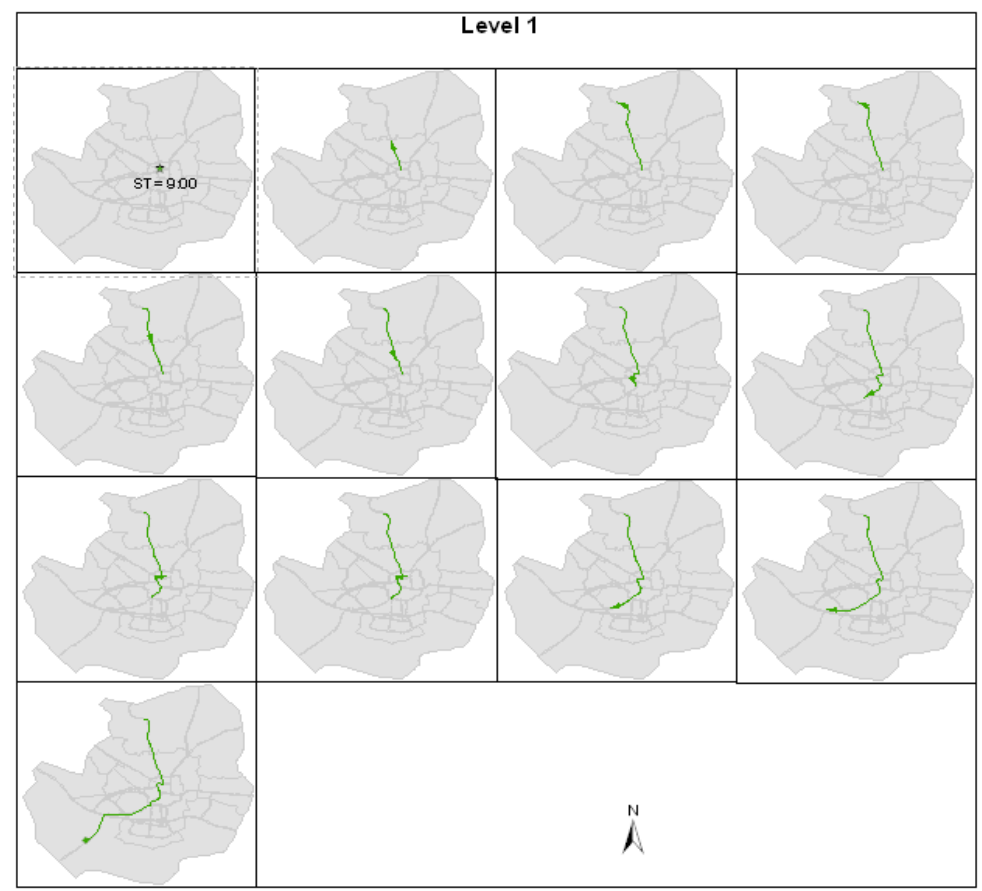

Figure 5. The first level of complexity for the multiple static maps with one moving object

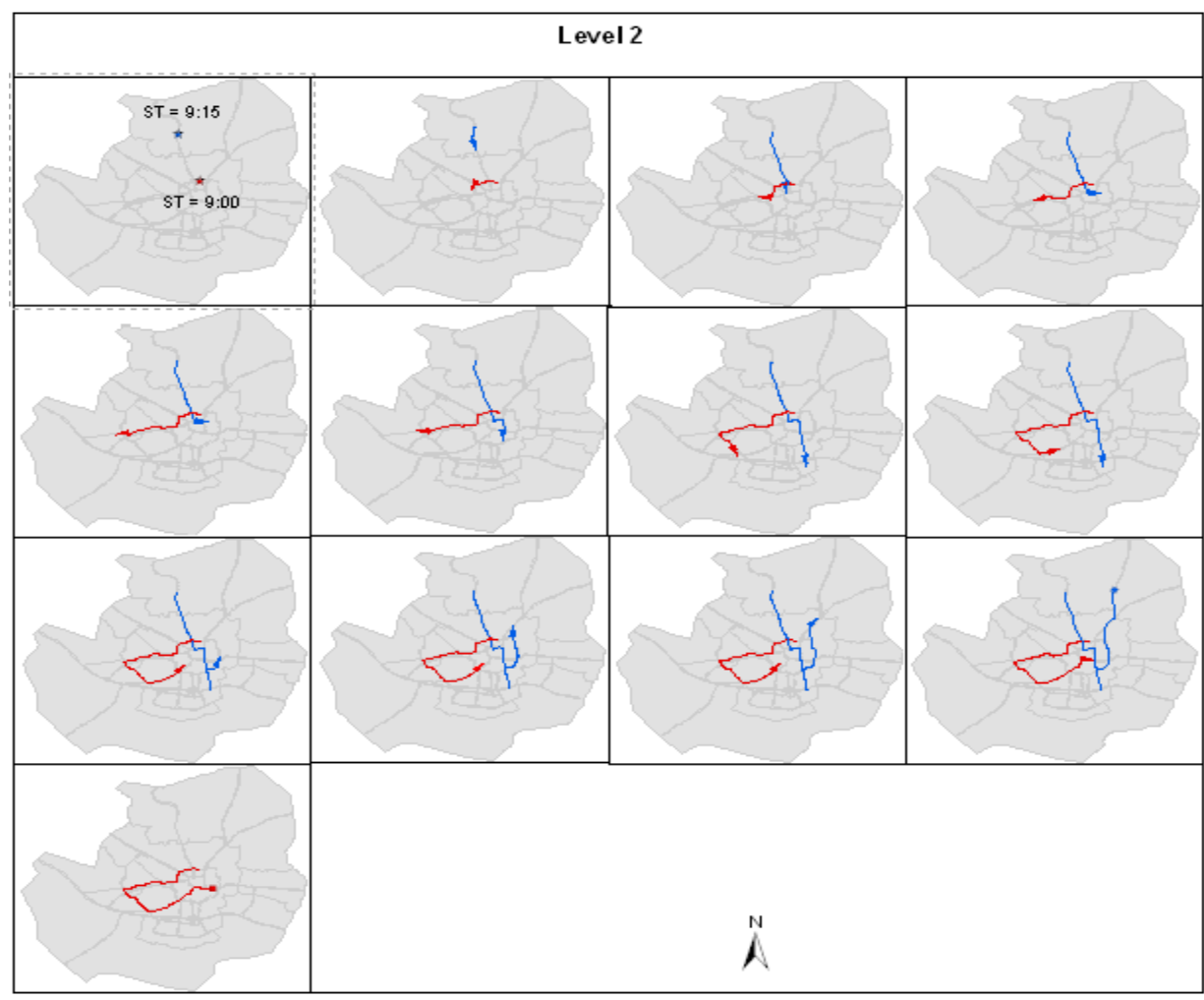

Figure 6. The second level of complexity for the multiple static maps with two moving objects 


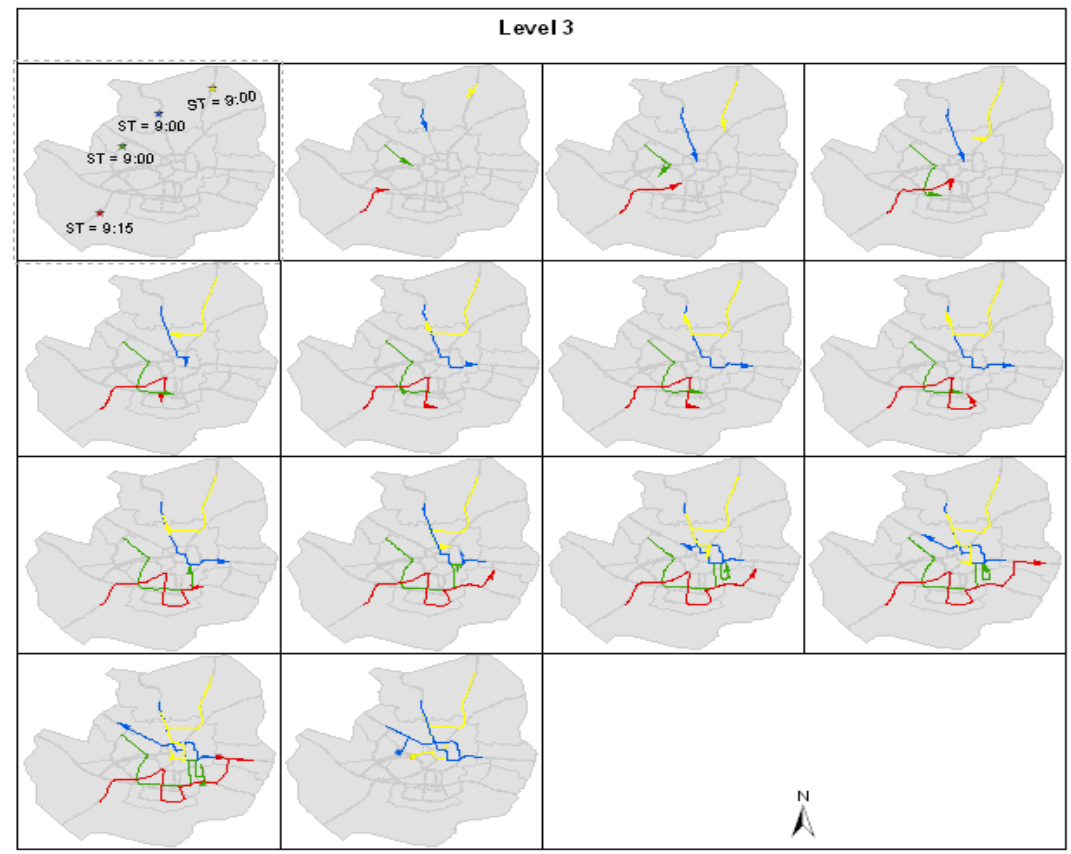

Figure 7. The third level of complexity for the multiple static maps with four moving objects

\subsubsection{Animation}

Like the single static and the multiple static maps, the animation is designed at three levels of complexity (see Figures $8 \mathrm{~A}, \mathrm{~B}$ and $\mathrm{C}$ ). The figures below show the display at the end of each animation. The animations have both the moving point and moving lines together. The lines show the path of the movement throughout the whole display. The point symbol for location used here is "dot” instead of an arrow. The minimum interaction environment is given (stop, pause, and rewind).

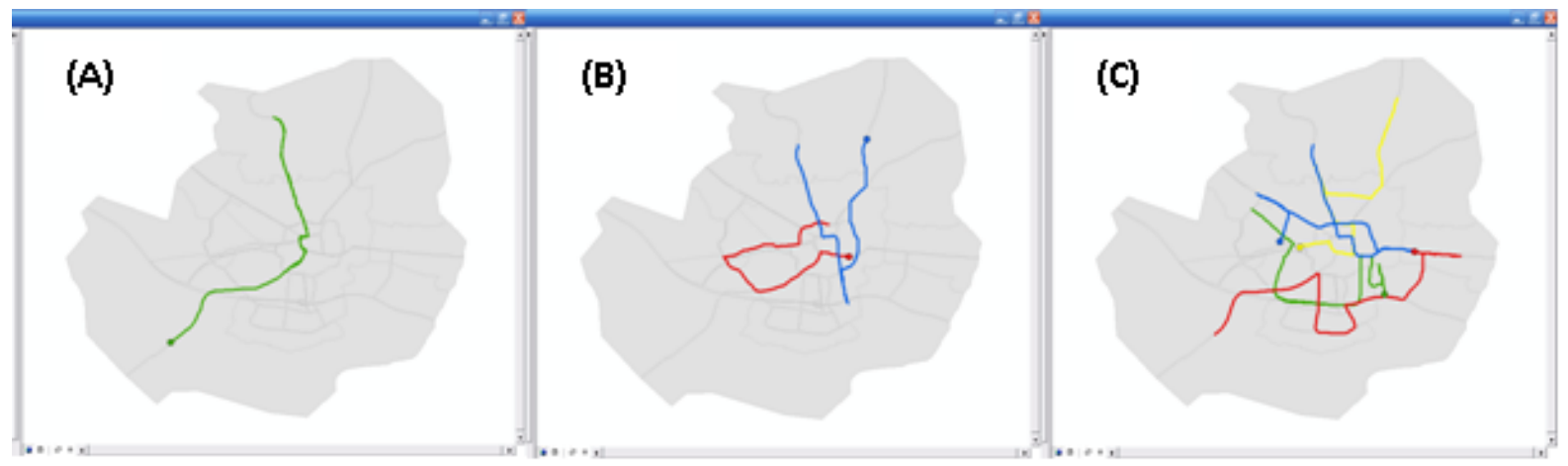

Figure 8. (A) The first level of complexity of the animation with one moving object; (B) the second level of complexity of the animation with two moving objects; and (C) the third level of complexity of the animation with four moving objects 


\subsubsection{The Space-time Cube}

Like all other representations, the space-time cube is designed in three levels of complexity (see Figures 9, 10 and 11). The background color of the space time cube is kept grey in order to have contrast with the objects (especially the main paths that move upward with time). The spatial coverage of the base map is shown on the (X, Y) axis while the time attribute is represented by the vertical axis (T). To clearly depict the path of movement, the foot print is displayed on the map. It shows the trajectory in space. Time is represented by the upward movement of the path from the start. Vertical lines that divide the space of the cube and horizontal lines across at every 15 minutes intervals are displayed as reference to locate objects in space and time.

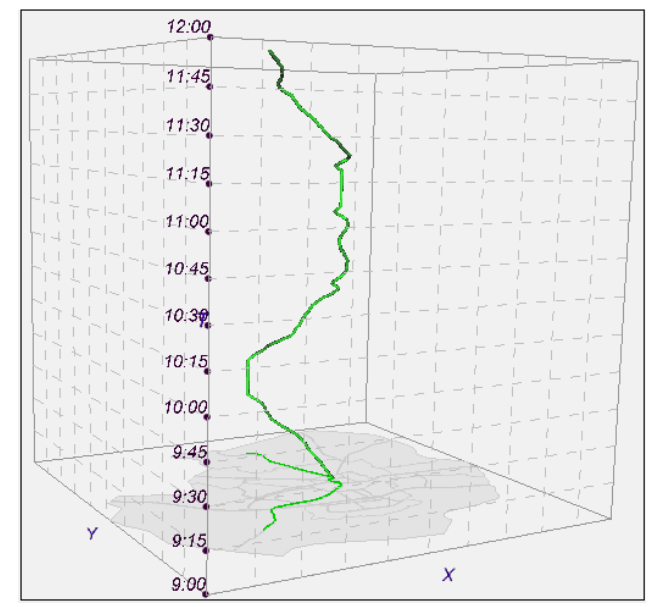

Figure 9. The first level of complexity for the space-time cube with one moving object.

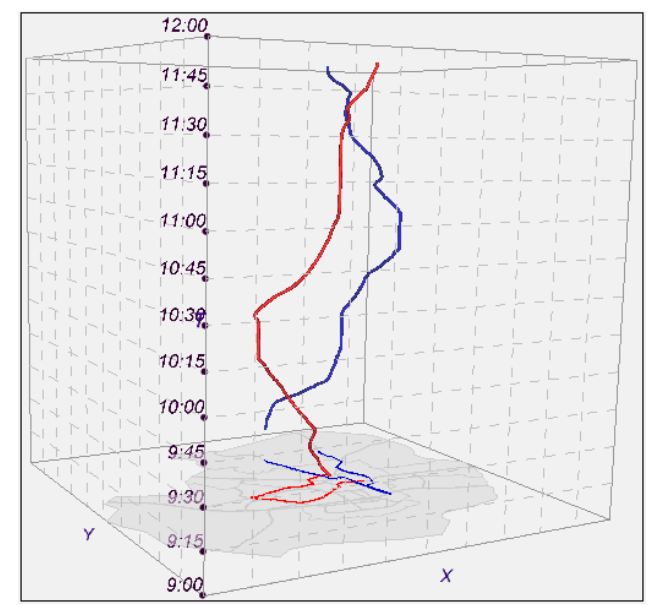

Figure 10. The second level of complexity for the space-time cube with two moving objects. 


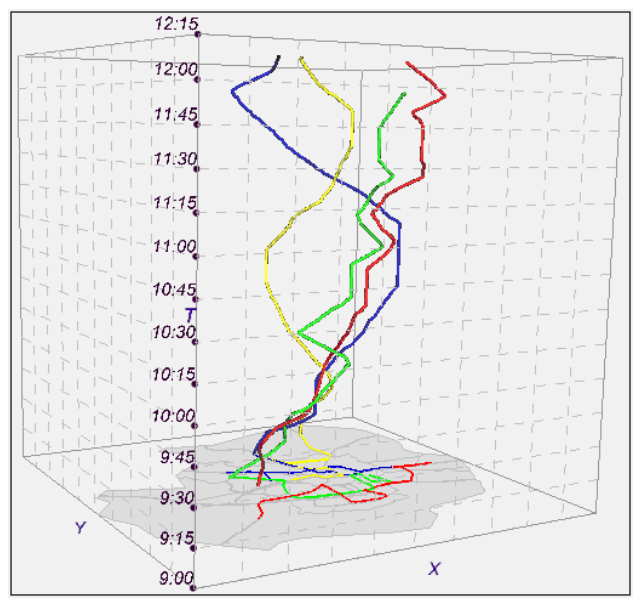

Figure 11. The third level of complexity for the space-time cube with four moving objects.

\subsection{Implementation of the Design}

The point data are developed in such ways that have the movement characteristics of walking people (stops, returns, speed change, and path). A series of segments is needed for the moving objects in the animation to show the development of the paths over time. This is done to enrich animation in visualizing the movement characteristics. The series of segments are created directly from the digitized point features. Points and segments are used for building both the static maps and the animation. For the paths of the single static maps, other line data are developed.

The time attribute is important since movement is a spatiotemporal phenomenon. A time attribute is added to both the point and the segment data. The time attribute of the line shape file is needed for the animation only. For the space-time cube the point data are used.

\section{6 . Measures of Evaluation}

In this research, the power of single static maps, multiple static maps, animation, and the spacetime cube in terms of representing some movement characteristics of walking people is tested against the three International Organization for Standardization (ISO) usability measuresefficiency, effectiveness and satisfaction.

In the context of conceptualizing and understanding the movement characteristics of the walking people represented in this research, the following questions can be asked to give context definitions for the measures of usability. 
- Do the representation methods clearly depict the aspects of the moving objects represented?

- Can the users clearly and easily conceptualize the aspects of the moving objects represented?

- Does it take the users' reasonable effort and time to conceptualize these aspects?

- Do the users feel comfortable with the representation methods used to visualize the selected aspects?

In this research, the first and second questions help to define effectiveness; the third question defines efficiency; and the fourth defines satisfaction. They are defined below.

- Effectiveness: whether or not the representation enables to execute the tasks correctly.

- Efficiency: Whether or not the representation enables to execute the tasks easily (with minimum effort) and quickly (with minimum time).

- Satisfaction: How acceptable is the representation in terms of representing moving objects (walking people).

\subsection{Empirical Testing}

\subsubsection{Test Methods}

Assessing the effectiveness and efficiency or investigating the satisfaction of the users with the representation methods is realized by selection of appropriate usability test methods. Some commonly used methods are questionnaires, interviews, focus group discussion and the think aloud method.

In this research, the questionnaire method (with users performing tasks) has been used. It is appropriate basically because it enables us to gather both objective data (correctness of answers) and subjective data that provide information about the three commonly used measures of usability (efficiency, effectiveness and satisfaction).

\subsubsection{Materials and Environment}

In the process of data entry, organization and analysis of two materials were made ready: the representations (in a computer) and the questionnaire document (in hard copy). The four representations (single static map, multiple static maps, animation, and the space-time cube) were stored in different folders in all the computers in the cluster that was used to conduct the 
evaluation session. Each folder contained the representations at three levels of complexity. The users opened the visualization required for the tasks section of the questionnaire. The real Space-Time Cube couldn't be used because it might create problems if users unintentionally use unnecessary function in the software. Failure in the functioning of the software may also happen and in this case it may need time to restart it. In this case, it might also need to work with the data to display the data in the space time cube again. Thus, it is prepared in a video version. This is done to give the participants the opportunity to see different views of the space-time cube to perform the tasks, to pause at a certain view and to continue to another view.

The questionnaire gives important notes first, like definitions of terms and the legend. Next, the tasks came, divided into three parts: part one included questions related to the conceptualization and understanding of the movement characteristics, part two holds questions related to the usability measures (effectiveness, efficiency, and satisfaction), and the third part questions about possible improvements of the visualizations. The experiment was conducted in one room with enough computers to work individually. In the session, discussion was not allowed. In case more explanation was needed, they raised their hand and ask the evaluator.

\subsubsection{Piloting and Sample Selection}

A selection of users who would understand the visualizations was considered essential. For this research, the geoinformatics MSc students (graduating class) were selected. But before the evaluation session with the students, a pilot test was conducted with two participants. The participants in the pilot test were students in other programs in ITC. From the pilot, it is managed to understand that it is not easy for students with relatively little experience with different visualizations to understand the representations. Thus, geoinformatics MSc students were selected as appropriate test participants because they gained the required level of knowledge about the visualizations during their study. The number of test participants was sixteen. These participants were placed in four groups with the intention to encounter each group with the four visualization methods at different levels of complexity, but each group was answering questions about another aspect of movement for a particular visualization method. This was done to reduce learning effects from one visualization method to the other and to enable the assessment of the impact on different levels of complexity. 


\subsubsection{Test Sessions}

The test session was held on 18 January 2008 at 10:40. It took 1:30 hours to finish the tasks. The visualizations were briefly introduced to inform and familiarize the participants. After explaining the objectives of the research and what aspects the participants are supposed to see, sample visualizations for a single static map, multiple static maps, and the space-time cube were shown by the experimenter. A sample animation was not prepared with the assumption that it is easy for the participants to understand it; thus, reduce the learning effect. After 15 minutes introduction, each user was provided with a questionnaire. Then completed questionnaires have been collected.

In the first part of the questionnaire, the participants were asked to answer questions about each aspect for a different visualization method, at various levels of complexity (see Table 1).

Table1. Number of respondents in each representation for each aspect at all levels of complexity. The same respondents answered at each level of the respective method and aspect.

\begin{tabular}{|lccccc|}
\hline \multicolumn{1}{|c}{ Aspects } & Single & Multiples & Animation & STC & Total \\
\hline Stops & & $\bullet 4$ & $\bullet 4$ & $\bullet 4$ & 12 \\
\hline Returns & $\bullet 4$ & $\bullet 4$ & $\bullet 4$ & $\diamond 4$ & 16 \\
\hline Speed change & $\bullet 4$ & $\diamond 4$ & $\bullet 4$ & $\bullet 4$ & 16 \\
\hline Path & $\bullet 4$ & $\bullet 4$ & $\diamond 4$ & $\bullet 4$ & 16 \\
\hline \multicolumn{1}{c}{ Total } & 12 & 16 & 16 & 16 & \\
\hline
\end{tabular}

Note: •-Group 1; $\odot$-Group 2; • -Group 3; $\diamond$-Group 4

The data collected were analyzed based on the percentage of respondents who gave correct answers given in the questionnaires (about the movement characteristics depicted in the representations). For the aspects which have two questions, the average percentage is taken.

In part two of the tasks in the questionnaire, users were asked to rate the effectiveness, efficiency, and satisfaction of the visualizations for the different aspects as low, moderate, and high. The participants also rated the visualizations at different levels of complexity. Since the purpose of this research is to find which method is most suitable for which aspect and at what level, only high ratings given by the users are used to determine the suitability. Low scores would probably mean low levels of acceptance by the users, which should be avoided in applications of the visualizations. 


\section{RESULTS AND DISCUSSION}

\subsection{Results}

\subsubsection{Conceptualizing Aspects of Moving Objects: Correctness of Answers}

The correctness of answers in each representation for each aspect at different level of complexity is analyzed in this part (Fig.12). The result shows that for stops the users answered most correctly in the space-time cube at Level 1, in multiple static maps at Level 2, and in animation at Level 3. For returns, correctness is the highest in single static map at Levels 1 and 2 and in animation at Level 3. For speed change the highest is in animation at Levels 1 and 3, and in animation and the single static map at Level 2 (their difference is less than 5\%).

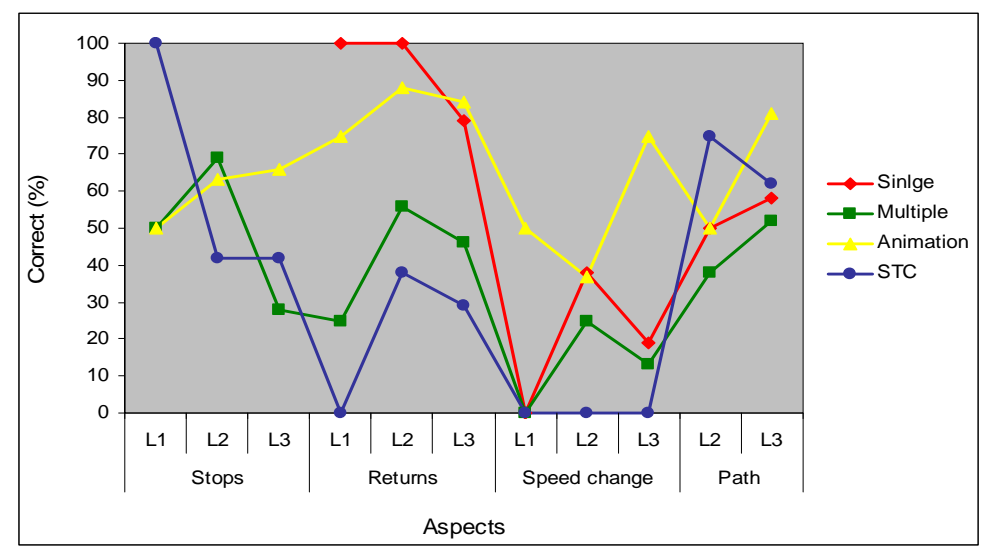

Figure 12. Correct answers (in \%) for each aspect at different levels of complexity of the visualizations

In depicting path, users answered most correctly in the space-time cube at Level 2 and in animation at Level 3. Extracting information about speed change seems the most challenging task. Single static and multiple static maps have zero percent at Level 1, while the space-time cube (STC) has zero percent at all levels of complexity. The space-time cube has also zero value for returns at Level 1.

\subsubsection{Impact of Level of Complexity and overall Result}

With respect to the impact of the level of complexity in the visualization, there is no uniform trend in the results (Figure 12). In some cases, the correctness is very low at Level 1 and high at a higher level; in other cases, it is the other way round. However, in some representations and for some aspects, there is an indication that the complexity influences the visualization. For example, it has influences in single static map in visualizing returns; and in the space-time cube in visualizing stops and path. 
The average of each level (Fig. 13) for each aspect, describes that for returns users answer most correctly with single static maps. Both animation and the space-time cube score best (having almost equal values, difference less than five percent) for stops, and path. Related to speed change, the participants answered most correctly using animation. Generally, the results in this part indicate that more than 50 percent of the respondents answered correctly using the animation for all aspects, while in the other representations it varies significantly from one aspect to another.

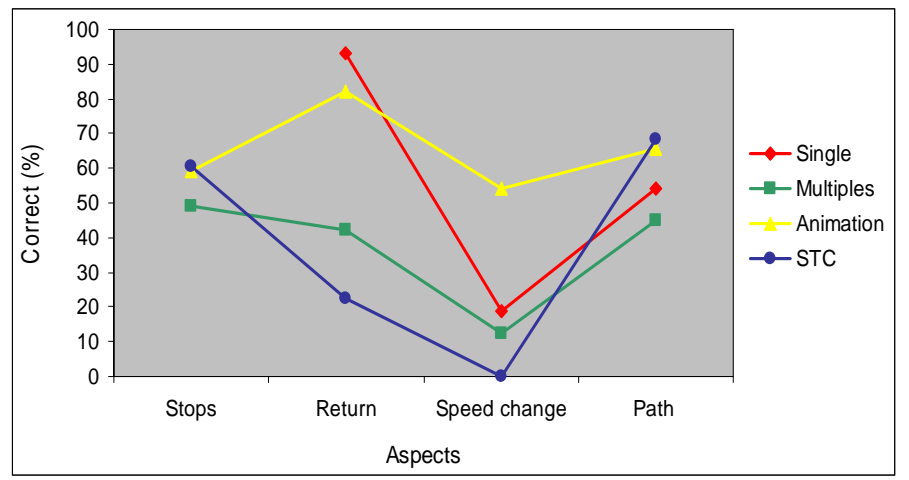

Figure 13. Correctness of answers in each representation for each aspect at all levels

\subsubsection{Usability Measures}

\subsubsection{Effectiveness}

The percentage of participants who gave "high" scores for the effectiveness is shown in figure 14. Animation scores the highest at Levels 1 and 3 for stops, at Level 2 for returns, and at Levels 1 and 2 for speed change. For the rest, it shares high scores with the other methods. The space-time cube has no high values in most of the aspects. The general result (the average of all levels in each aspect) shows that animation has got the highest effectiveness for all aspects.

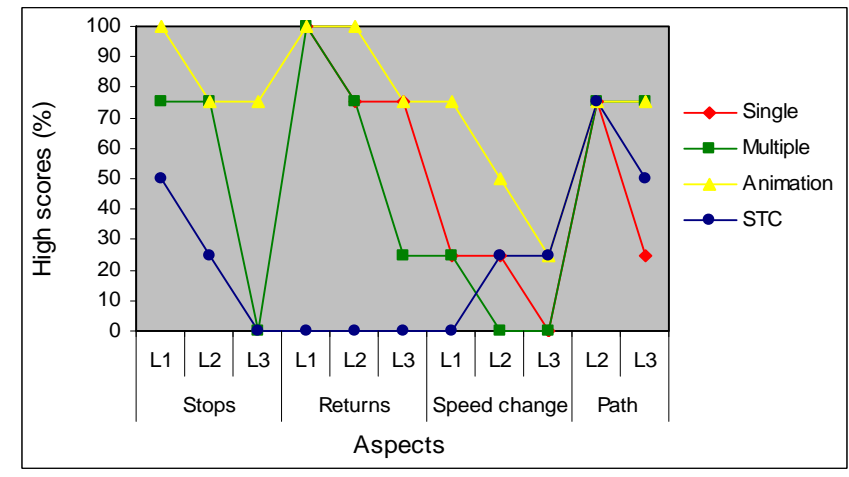

Figure 14. Percentage of "high" scores for the effectiveness of each representation (per aspect and level of complexity) 


\subsubsection{Efficiency}

The percentage of high scores for the efficiency of the representations is shown in figure 15. The scores of animation are highest at all levels for stops, at Levels 2 and 3 for returns, at Level 1 for speed change, and at Level 3 for path. It has equal efficiency scores with multiple static maps at Level 1 for returns, and with all the methods at Level 2 for path. It has no high scores for efficiency at Levels 2 and 3 for speed change. The space-time cube and single static maps lack more high scores than the other methods. Generally (taking the average of all levels in each aspect), animation is the most efficient method, especially for stops, returns, and path.

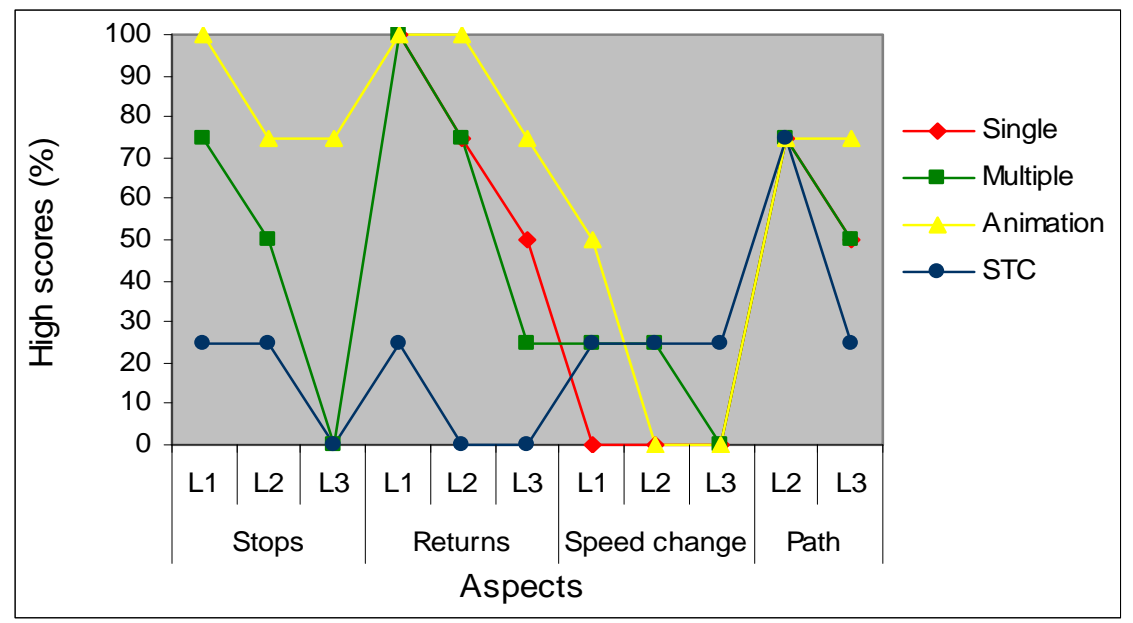

Figure 15. Percentage of "high" scores for the efficiency of each representation (per aspect and level of complexity).

\subsubsection{Satisfaction}

The percentage of high scores for satisfaction and the visualizations given to the users is illustrated in figure 16. Animation has highest scores at Levels 1 and 3 for stops, at all the levels for returns and at Level 1 for speed change, while it has no high scores at Levels 2 and 3 for speed change. The other methods lack more high scores for different aspects. Thus, animation gives greatest satisfaction to users (taking the average of all levels in each aspect), especially for stops and returns. 


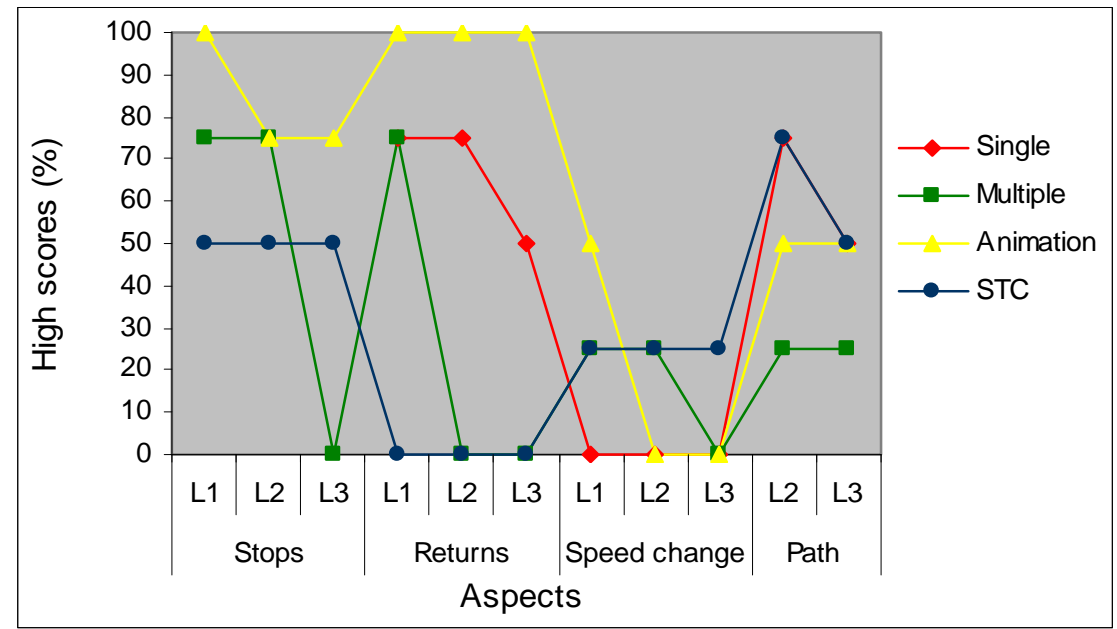

Figure 16. Percentage of "high" scores for the satisfaction of each representation (per aspect and level of complexity).

\subsubsection{Impact of Level of Complexity on the Usability of the Visualizations and overall Result}

The overall usability of the representations varies with the level of complexity (Fig. 17). In each representation, the percentage of the high scores is large at Level 1 and descends towards Level 3. However, the situation for the space-time cube for speed change is an exception; here it ascends from Level 1 to Level 2. This might be because users have learned from the lower level and in effect got a better impression in higher level to perceive and understand speed change in the space-time cube.

The results at each level of complexity show that animation is the most usable at all levels of complexity for visualizing stops and returns, at Level 3 for path, and at Level 1 for speed change. The space-time cube is the most usable for speed change at Levels 2 and 3 and for path at Level 2. 


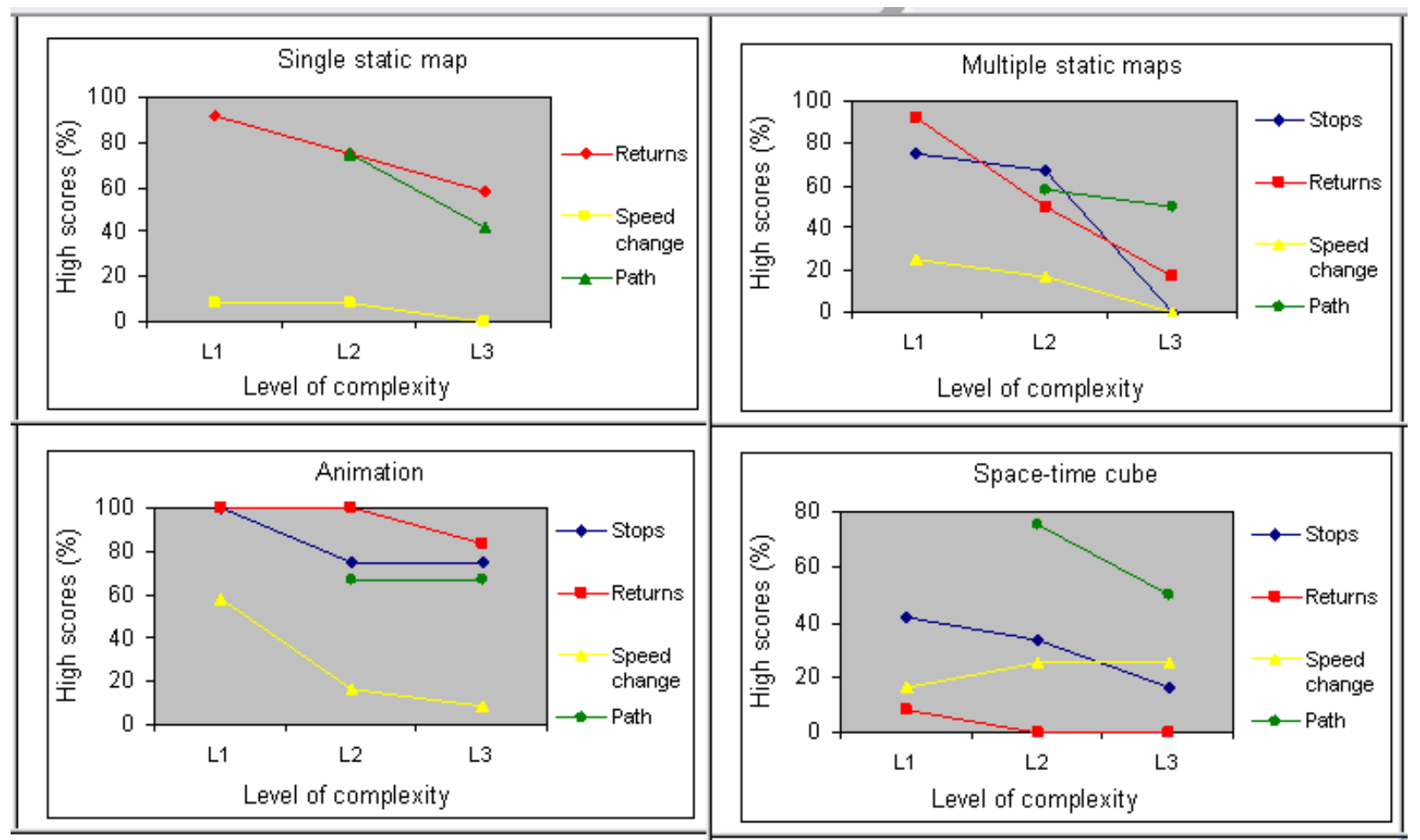

Figure 17. The impact of the level of complexity in the visualization for each aspect

The overall result (the average of high ratings of all levels for the usability measures: effectiveness, efficiency and satisfaction-regardless of the level of complexity) (Fig. 18) describes that animation is the most usable method for all aspects. Another important depiction is that the usability of the space-time cube is very low for visualizing returns as compared to the other methods.

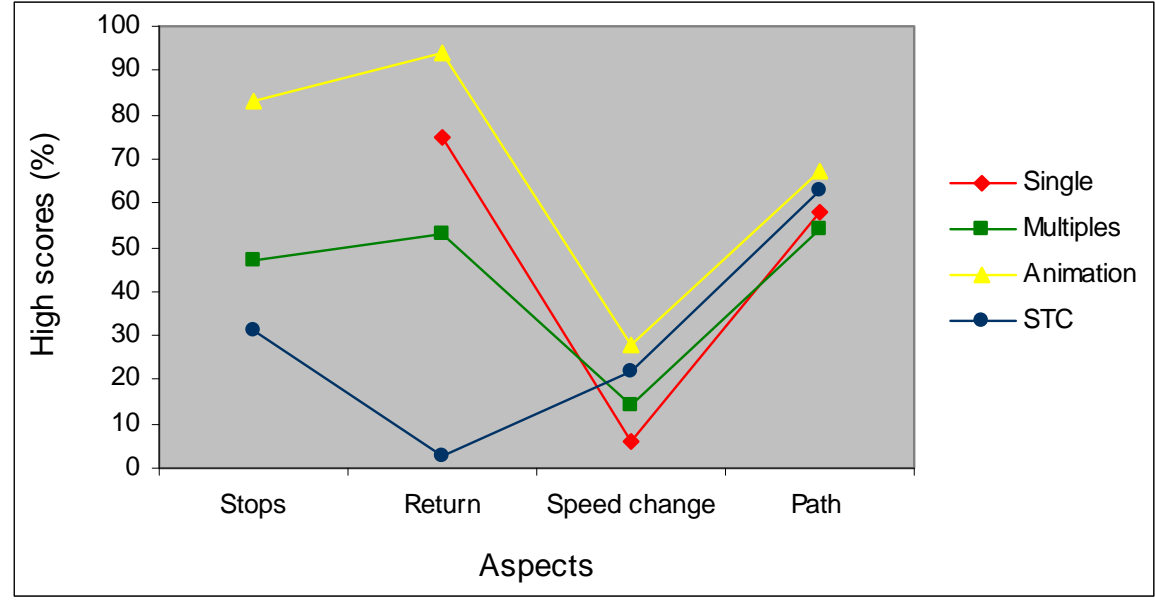

Figure 18. Overall usability taking the average of high scores of all levels in all usability measures (effectiveness, efficiency, and satisfaction) 


\subsubsection{Adjusted Framework}

From the literature, knowledge and common sense, a framework (predicted) as to the suitability of the visualizations for showing returns, stops, speed change, and path at three levels of complexity (Table 2) was developed. An adjusted framework is developed after test and analysis. For the purpose of adjusting the framework (Table 3), both the correctness and usability measures are considered. The percentages in correctness and usability at each level are added and their average percentage is taken. The resulting percentages are translated into three classes as shown below:

- $0-<50=$ low

- $\quad 50-<75=$ moderate

- $\quad 75-100$ = high

According to the adjusted framework, animation has high suitability for stops at Level 1, and at all levels for returns. For the visualization of path, the space-time cube has high suitability at Level 1. Space -time cube is the most suitable in depicting path may be because the main paths are accompanied by the footprints. Overall, the framework shows that animation is the most suitable method.

Table 2. Predicted suitability of the representations at three levels of complexity: from low (Level 1) to high (Level 3). The predicted suitability is represented by tints from light (low) to dark (high). Stops are not tested for single static maps. Since the questions related to path needed more than two objects, Level 1 is skipped.

\begin{tabular}{|c|c|c|c|c|c|c|c|c|c|c|c|}
\hline \multirow[b]{3}{*}{ Methods } & \multicolumn{3}{|c|}{ Stops } & & Retu & & \multicolumn{3}{|c|}{$\begin{array}{c}\text { Speed } \\
\text { change }\end{array}$} & \multicolumn{2}{|c|}{ Path } \\
\hline & \multicolumn{11}{|c|}{ Complexities } \\
\hline & L1 & $\mathrm{L} 2$ & L3 & L1 & $\mathrm{L} 2$ & L3 & L1 & L2 & L3 & $\mathrm{L} 2$ & L3 \\
\hline Single & NA & NA & NA & & & & & & & & \\
\hline Multiple & & & & & & & & & & & \\
\hline Animation & & & & & & & & & & & \\
\hline STC & & & & & & & & & & & \\
\hline
\end{tabular}

Note: NA= Not Applicable; L1, L2, L3=levels of complexity. 
Table 3. Adjusted framework from the results found out in 3.1.2.

\begin{tabular}{|l|l|l|l|l|l|l|l|l|l|l|l|}
\hline & \multicolumn{9}{|c|}{ Stops } & \multicolumn{9}{c|}{ Returns } & \multicolumn{3}{c|}{$\begin{array}{c}\text { Speed } \\
\text { change }\end{array}$} & \multicolumn{2}{c|}{ Path } \\
\cline { 2 - 12 } Methods & L1 & L2 & L3 & L1 & L2 & L3 & L1 & L2 & L3 & L2 & L3 \\
\hline Single & NA & NA & NA & & & & & & & & \\
\hline Multiple & & & & & & & & & & & \\
\hline Animation & & & & & & & & & & & \\
\hline STC & & & & & & & & & & & \\
\hline
\end{tabular}

Note: NA= Not Applicable; L1, L2, L3=levels of complexity.

\subsection{DISCUSSION}

The results found (in part 3.1.1 about the conceptualization (correctness) and in 3.1.2 about the subjective usability) are different. In conceptualization, the level of complexity has no influence on the perception and understanding of users. Sometimes, the percentage of correctness increases with the level of complexity, other times it decreases. Increase may be because the users learn at the lower level and when they come to the next higher level, they perceive and understand things better. So there may be a learning effect even though the participants saw different trajectories at each level of a representation.

The subjective suitability results clearly show that the level of complexity matters, as expected. The level of complexity had an impact on the effectiveness and efficiency of the methods, and on the satisfaction of the users.

In the two results, however, given the difference, the general situation reflects that animation is the most suitable representation because it has got overall higher scores in both results (regardless of the levels of complexity and the aspects). An important thing in animation is that it has no high scores in efficiency and satisfaction for the visualization of speed change at Levels 2 and 3 (even in the correctness and effectiveness measures, its value for speed change is the lowest of all other aspects). This implies that perceiving change in speed in animation is difficult at high complexities.

For the purpose of adjusting the framework both the results in the correctness and the subjective usability are considered. Thus, according to the framework, animation is the most suitable representation, except that the space time cube is more suitable for showing path at Level 2. That animation is the most suitable may be because it shows real dynamics and that makes it 
easier for users to perceive and understand the aspects.

The predicted framework and the adjusted one have some similarities and differences. The two frameworks are similar for multiple static maps for all aspects except for path at Level 2. That is, the prediction almost fits the results found after assessment. There are bigger differences for the space-time cube than for the other representations. The suitability of the space-time cube is lower than expected. This might be because of two reasons: 1) the space-time cube requires higher expertise than the other representations. 2) The space-time cube perhaps needs the real interactive interface. If we see the most suitable representation (animation), the predicted and the resulting suitability are similar for visualizing stops at Levels 1 and 3, returns at Levels 1 and 2, and path at Level 3. In the rest, they show differences. Generally, the two frameworks show that animation is the most suitable representation.

\section{CONCLUSION}

The main goal of this research was to find the optimal visualization for moving objects (for an application involving walking people). Moving objects exhibit various behaviors, among which their movement behavior was the concern of this research. This research was about investigating how users can understand and acquire geospatial knowledge about moving people from cartographic representations (static maps, animation, and the space-time cube). In the course of investigating the optimal representation, there is the issue of comparability. Many researches make comparisons of these representations to find which one is more effective for a particular use. Some contend that comparability should be taken into account so as to come to the deduction that one is more effective than the other.

In this research, comparability has not been an important issue. Finding the optimal representation is made without the demand that all the representations should be exactly the same in design. Except for the stops in single static maps, however, the information that could be extracted is comparable in each map type, while leaving the potential power of each representation unaffected. A minimum interactive environment is given to animation and the video version of the space-time cube to enable the users to stop, pause, and rewind. So, when trying to find the optimal representation (for example, for moving objects), the possible potential of the representations is maintained. In effect, in this research, every possibility to exploit the potential of the representations is undertaken. For example, animation is accompanied by 
growing path and the space-time cube is prepared with foot prints, grids for space and time indication, and it is also provided to users as a video to enable the users to see different views. Thus, whether these representations are comparable with the static maps is not of prime importance.

After making the assessments in this research, overall, it is found out that animation is the optimal representation for most aspects (stops, returns and speed change) of moving objects (for walking people application). It has been easier for users of animation to conceive and understand the movement characteristics (stops, returns, speed change, and path) than other representations. It might be because dynamics can more easily and lively be seen in animation than in the other representations. That is, the real dynamics of the objects can be seen (represented) in animation. The result of this research can also be used for other applications, such as the visualization of moving cars for visualizing traffic situations, visualizing clouds for meteorological application, visualizing migration of birds (without considering altitude), and hurricanes to visualize their movement (in which speed, direction change, etc can be seen.

\section{REFERENCES}

Andrienko, N \& Andrienko. 2007. Designing visual analytics methods for massive collections of movement data. Cartographica: the international journal for geographic information and geovisualization, 42(2): 117-138.

Blok, C. A. 2005. Dynamic visualization variables in animation to support monitoring of spatial phenomena. Nederlandse Geografische Studies, Netherlands Geographical Studies; 328. Utrecht, Enschede, Universiteit Utrecht, ITC: 188.

de By, R.A., Georgiadou, P.Y., Knippers, R.A., Kraak, M.J., Sun, Y., Weir, M.J.C \& van Westen, C.J. 2004. Principles of geographic information systems: an introductory textbook. Enschede, ITC, 2004. ITC Educational Textbook Series 1, ISBN: 90-6164-226-4, 226 pp.

Dorling, D \& Openshaw, S. 1994. Using computer animation to visualize space - time patterns. Environment and Planning B. Planning and Design, 19(2): 639-650.

Fabrikant, S. I. 2005. Towards an understanding of geo-visualization with dynamic displays: issues and prospects. Proceedings, American Association for Artificial Intelligence (AAAI) 2005 Springer Symposium Series: Reasoning with Mental and External 
Diagrams: Computational Modelling and Spatial Assistance, Stanford University, Stanford, CA, March 21-23, 2005: 6-11.

Fabrikant, S. I \& Goldsberry, K. 2005. Thematic relevance and perceptual salience of dynamic geo-visualization displays. Proceedings, 22nd ICA/ACI International Cartographic Conference, A Coruna, Spain, Jul. 9-16, 2005.

Karl, D. 1992. Cartographic animation : potential and research issues. In: Cartographic Perspectives, 13(1992)fall, pp. 3-9.

Kim, S., Yoon, M., Whang, S. M., Tversky, B \& Morrison, J. B. 2007. The effect of animation on comprehension and interest. Journal of Computer Assisted Learning, 23(3): 260-270.

Kraak, M. J. 1999. Cartography and the use of animation. In: Multimedia in cartography / W. Cartwright, M. Peterson and G. Gartner: 1999. pp. 173-180.

Kraak, M. J. 2003. Space - time cube revisited from a geovisualization perspective. In: ICC 2003 : Proceedings of the 21st international cartographic conference : cartographic renaissance, 10-16 August 2003, Durban, South Africa. International Cartographic Association (ICA): 2003. pp. 1988-1996.

Kraak, M. J \& Koussoulakou, A. 2004. Visualization environment for the space - time cube. In: SDH 2004 : Proceedings of the 11th international symposium on spatial data handling : advances in spatial data handling II. : 23-25 August 2004, University of Leichester. / ed. by P.F. Fisher. - Berlin etc.: Springer, 2004. pp. 189-200.

Kraak, M. J \& Ormeling, F.J. 2003. Cartography: visualization of geospatial data. Harlow, Addison Wesley Longman.

Kristensson, P.O., Ack, N.D., Anundi, D., Ornstad, M.B., Gillberg, H., Haraldsson, J., M_artensson, I., Nordvall, M \& Jose_nSt_ahl. 2007. The trade-offs with space time cube representation of spatiotemporal patterns, Department of Computer and Information Science, Linkoping University, Sweden, November 2, 2007.

Krueger, R. A \& Casey, M.A. 2000. Focus groups: a practical guide for applied research. Sage Publications, Inc.

Kumar, R. 2005. Research methodology : a step - by - step guide for beginners. Sage, London.

Maratnia, N \& de By, R.A. 2004. Spatiotemporal compression techniques for moving point objects. In: Advances in database technology - EDBT 2004: proceedings of the $9^{\text {th }}$ international conference on extending database technology, Heraklion, Crete, Greece, 
March 14-18, 2004. / editor E. Bertino, S. Christodoulakis, D. Plexousakis, V. Christophides, M. Koubarakis, K. Bóhm, E. Ferrari. - Berlin etc.: Springer, 2004. (Lecture Notes in Computer Science: 2992) pp. 765-782.

Morgan, D \& Krueger, R.A.1997. The focus group kit, Sage Publications, Inc.

Nielsen, J. 1993. Usability engineering. Boston, Academic Press.

Ogao, P. J. 2002. Exploratory visualization of temporal geospatial data using animation. ITC Dissertation, 89. Utrecht, Utrecht University: 158.

Opach, T. 2007. An experimental research of effectiveness of temporal animated maps. XXIII International Cartographic Conference. 4-10 August 2007, Moscow, Russia, CD-ROM, 13pp.

Slocum, T. A. 1999. Thematic cartography and visualization. Upper Saddle River, Prentice Hall.

Tversky, B., Morrison, J. B \& Betrancourt, M. 2002. "Animation: can it facilitate?" International Journal of Human-Computer Studies 57(4): 247-262.

Weghe, N.V., Cohn, A.G., Bogaert, P \& Maeyer, P.D. 2004. Representations of moving objects along a road network Geoinformatics 2004: Proceeding of $12^{\text {th }}$ International Conference on Geoinformatics - geospatial Information Research: Bridging the Pacific and Atlantic University of Gavle Sweden, 7-9 June 2004.

Xia, L \& Kraak, M.J. 2005. New views on multivariable spatio-temporal data: the space time cube expanded. In: ISPRS STM 2005: proceedings of the international symposium on spatio-temporal modelling, spatial reasoning, analysis, data mining and fusion: ISSTM, August 27-29 2005, Peking University, China. Vol. XXXVI, part 2/W25. pp. 199-201. 